\title{
Structural Perceptrons for Attributed Graphs
}

\author{
Brijnesh J. Jain and Fritz Wysotzki \\ Dept. of Electrical Engineering and Computer Science \\ Technical University Berlin, Germany
}

\begin{abstract}
We propose a structural perceptron for supervised and unsupervised learning on data represented in terms of attributed graphs. We integrate structural perceptrons into a multi-layer perceptron and competitive learning network to provide examples of supervised and unsupervised neural learning machines which are suited to process graphs. In first experiments the proposed algorithms were successfully applied to function regression, classification, and clustering.
\end{abstract}

\section{Introduction}

It is common practice to represent data in terms of vectors of an Euclidean space, because the Euclidean space provides powerful analytical techniques for data analysis usually not available in other representations. Such a representation, however, is too limited for many relevant application areas including domains such as computer vision, bioinformatics, chemistry, or text mining. A more versatile and expressive tool for representing structured data are, for example, attributed graphs.

Despite its applicability and importance, learning on graphs is still widely unexplored. Current research focus on (1) the problem of embedding graphs into vector spaces to access the whole plethora of analytical tools (e.g. [10]); (2) algorithms on pairwise proximity data [6]; (3) kernel methods for structures [2]; and (4) adaptive processing of graphs (e.g. $[1,11,12])$. In the structural pattern recognition literature, adaptive processing of graphs is mainly concerned with devising clustering algorithms (e.g. [1,4,9]). In the neural networks community, connectionist models have been proposed for supervised learning on the rather restrictive class of directed (ordered) acyclic graphs [12].

In this contribution, we suggest a structural perceptron for adaptive processing of graphs within a supervised and unsupervised setting. To facilitate adaptive processing, we associate a structural perceptron with an attributed weight graph and replace the concept of an inner product of vectors by the Schur-Hadamard (SH) inner product of graphs. Despite its name, the SH inner product is not an inner product, but shares some useful properties of an inner product to extend supervised and unsupervised neural learning machines for attributed graphs. Finally, (un)supervised training of networks composed of structural units is then based on minimizing a suitable error function as a function of adjustable weights.

The rest of this paper is organized as follows: Section 2 introduces the SH inner product. Section 3 describes structural neural learning machines. In Section 4 we present first experiments. Finally, Section 5 concludes. 


\section{The Schur-Hadamard Inner Product}

This section provides basic notions and introduces the SH inner product.

Terminology. Let $\mathcal{S}$ be a set. By $\mathcal{S}^{[2]}$ we denote the set of all ordered tuples $(i, j) \in \mathcal{S}^{2}$ with $i \neq j$. The set of all $n \times m$-matrices $A=\left(\boldsymbol{a}_{i j}\right)$ with entries $\boldsymbol{a}_{i j}$ from a set $\mathcal{S}$ is denoted by $\mathcal{M}_{n \times m}(\mathcal{S})$.

Let $\mathcal{A}$ be an inner product space over $\mathbb{R}$, for example $\mathcal{A}=\mathbb{R}^{m}$. An attributed graph is a tuple $X=(V, \mu)$ consisting of a finite set $V \neq \emptyset$ and a function $\mu: V^{2} \rightarrow \mathcal{A}$. The elements of $V$ are the vertices of the graph $X$ and the pairs $(i, j) \in V^{[2]}$ with $\mu(i, j) \neq \mathbf{0}$ are its edges. The function $\mu$ is the attribute function of $X$. By $\mathcal{G}_{\mathcal{A}}$ we denote the set of attributed graphs with attributes from $\mathcal{A}$. The vertex set of a graph $X$ is referred to as $V(X)$, its edge set as $E(X)$, and its attribute function as $\mu_{X}$. Let $X$ be an attributed graph of order $|X|=|V(X)|=$ $n$. The (attributed) adjacency matrix of $X$ is a matrix $A(X)=\left(\boldsymbol{x}_{i j}\right) \in \mathcal{M}_{n \times n}(\mathcal{A})$ with entries $\boldsymbol{x}_{i j}=\mu_{X}(i, j)$.

A permutation acting on $X$ is a bijection $\pi: V(X) \rightarrow V(X)$ from $V(X)$ onto itself. The image graph of a permutation $\pi$ acting on $X$ is denoted by $X^{\pi}$. The set $\mathcal{S}_{X}$ of all permutations acting on $X$ is called the symmetric group of $X$. Note that in general $A(X) \neq A\left(X^{\pi}\right)$.

The Schur-Hadamard Inner Product. First we define the inner product of attributed matrices. Let $A, B \in \mathcal{M}_{n \times n}(\mathcal{A})$ be matrices with $A=\left(\boldsymbol{a}_{i j}\right)$ and $B=$ $\left(\boldsymbol{b}_{i j}\right)$. Addition of matrices and scalar multiplication are defined componentwise. The inner product $\langle$,$\rangle defined on \mathcal{A}$ induces an inner product on $\mathcal{M}_{n \times n}(\mathcal{A})$ by

$$
\langle A, B\rangle=\sum_{i=1}^{n} \sum_{j=i}^{n}\left\langle\boldsymbol{a}_{i j}, \boldsymbol{b}_{i j}\right\rangle .
$$

To define a formal addition, scalar multiplication, and the SH inner product of graphs we use the following technical convention: Let $X, Y \in \mathcal{G}_{\mathcal{A}}$. Suppose that $n=\max \{|X|,|Y|\}$ and $m=\min \{|X|,|Y|\}$. We implicitly assume that both graphs are of the same order $n$ by inserting $n-m$ isolated nodes into the smaller of both graphs, each labeled with $\mathbf{0}$. Then $X+Y=A(X)+A(Y)$ and $\lambda X=\lambda A(X)$ for all $\lambda \in \mathbb{R}$. The SH inner product is of the form

$$
\sigma: \mathcal{G}_{\mathcal{A}} \times \mathcal{G}_{\mathcal{A}} \rightarrow \mathbb{R}, \quad(X, Y) \mapsto \max _{\pi \in S_{X}}\left\langle A\left(X^{\pi}\right), A(Y)\right\rangle .
$$

A permutation $\pi \in \mathcal{S}_{X}$ with $\sigma(X, Y)=\left\langle A\left(X^{\pi}\right), A(Y)\right\rangle$ is called embedding from $X$ into $Y$. By $\mathcal{I}(X, Y)$ we denote the set of all embeddings from $X$ into $Y$.

In [7] it is shown that the SH inner product is symmetric and positive, but not bilinear. Hence, it is not an inner product. But the Cauchy-Schwarz inequality holds giving rise to an Euclidean metric $\delta($.) induced by the SH inner product

$$
\delta: \mathcal{G}_{\mathcal{A}} \times \mathcal{G}_{\mathcal{A}} \rightarrow \mathbb{R}, \quad(X, Y) \mapsto \sqrt{\sigma(X, X)-2 \sigma(X, Y)+\sigma(Y, Y)} .
$$


We conclude this section with two important remarks: (1) Determining the SH inner product is NP complete, since it generalizes the maximum common subgraph problem. (2) There is no restriction on the order of the graphs. Aligning two graphs to the same size is a pure technical trick and is not required in a practical implementation when computing the SH inner product.

\section{$3 \quad$ Structural Perceptrons}

This section first introduces structural perceptrons for attributed graphs. Next we provide an example of a supervised and of an unsupervised neural learning machine. Finally, we discuss some limitations of this approach.

The Model. Let $X, W \in \mathcal{G}_{\mathcal{A}}$ be attributed graphs with adjacency matrix $A(X)=\left(\boldsymbol{x}_{i j}\right)$ and $A(W)=\left(\boldsymbol{w}_{i j}\right)$. A structural perceptron is of the form:

$$
\begin{aligned}
& a=\sigma(X, W)+b \\
& y=g(a)
\end{aligned}
$$

where $X$ denotes a data graph, $W$ is the weight matrix of the perceptron, $b$ is the bias, $\sigma(X, W)$ the SH inner product of $X$ and $W, a$ is the activation, $g($.$) is$ the non-linear activation function, and $y$ is the output.

Supervised Learning with Multi-layer Perceptrons. Assume that we are given a training sample $\mathcal{Z}=(\mathcal{X}, \mathcal{Y})$ consisting of a set of data graphs $\mathcal{X}=\left\{X_{1}, \ldots, X_{M}\right\} \subseteq \mathcal{G}_{\mathcal{A}}$ together with corresponding output values $\mathcal{Y}=$ $\left\{\hat{\boldsymbol{y}}_{1}, \ldots, \hat{\boldsymbol{y}}_{M}\right\} \subseteq \mathbb{R}^{m}$. The problem is to estimate an unknown relation

$$
f: \mathcal{G}_{\mathcal{A}} \rightarrow \mathbb{R}^{m}
$$

given the sample $\mathcal{Z}$ and a set $\mathcal{H}$ of functions $h: \mathcal{G}_{\mathcal{A}} \rightarrow \mathbb{R}^{m}$. Here we are concerned with functions $h \in \mathcal{H}$ implemented by MLPs for attributed graphs. The functions of $\mathcal{H}$ are of the form $h(X, \mathcal{W})=h_{L}\left(\boldsymbol{y}_{L-1}, \mathcal{W}_{L}\right)$ with

$$
\begin{aligned}
& \boldsymbol{y}_{\mathbf{1}}=h_{1}\left(X, \mathcal{W}_{l}\right) \\
& \boldsymbol{y}_{\boldsymbol{l}}=h_{l}\left(\boldsymbol{y}_{\boldsymbol{l}-\mathbf{1}}, \mathcal{W}_{l}\right)
\end{aligned}
$$

for all layers $l \in\{1, \ldots, L\}$ where $X$ is a data graph, $\boldsymbol{y}_{\boldsymbol{l}}$ is a vector representing the output of the function $h_{l}$, and $\mathcal{W}_{l}$ is the set of adjustable weights (including biases) of $h_{l}$. The learning task considered here is based on minimizing a suitable error function with respect to the weights and biases by gradient descent using the back-propagation algorithm.

We commence with supervised learning of a single structural perceptron using the error-back-propagation algorithm. Since $L=m=1$, we may drop dispensable indices. For any data graph $X \in \mathcal{X}$ let $E=E(y)$ be a differentiable error function of the network output variables. The derivative of $E$ with respect to some weight $w_{i j}^{k} \in \mathcal{W}$ is of the form

$$
\frac{\partial E}{\partial w_{i j}^{k}}=\frac{\partial E}{\partial a} \frac{\partial a}{\partial w_{i j}^{k}}=\delta \frac{\partial a}{\partial w_{i j}^{k}}
$$

where $w_{i j}^{k}$ is the $k^{\text {th }}$ component of the vector $\boldsymbol{w}_{i j} \in \mathcal{A}$ and 


$$
\delta=\frac{\partial E}{\partial a}=g^{\prime}(a) \frac{\partial E}{\partial y}
$$

is often referred to as the error. Since $g$ and $E$ are known we substitute appropriate expressions for $g^{\prime}(a)$ and $\partial E / \partial y$ to evaluate (3). To evaluate $\partial a / \partial w_{i j}^{k}$ we choose an embedding $\pi \in \mathcal{I}(X, W)$ and set

$$
\frac{\partial a}{\partial w_{i j}^{k}}=\frac{\partial}{\partial w_{i j}^{k}}\{\sigma(X, W)+b\}=\frac{\partial}{\partial w_{i j}^{k}}\left\{\left\langle A\left(X^{\pi}\right), A(W)\right\rangle+b\right\}=\pi\left(x_{i j}^{k}\right)
$$

where $\pi\left(x_{i j}^{k}\right)=x_{r s}^{k}$ with $r=i^{\pi}$ and $s=j^{\pi}$. Putting all together we obtain

$$
\frac{\partial E}{\partial w_{i j}^{k}}=\delta \cdot \pi\left(x_{i j}^{k}\right)
$$

Since the bias $b$ is not involved in the computation of the SH inner product, the derivative $\partial E / \partial b=\delta$ is of the same form as in the case of vectorial perceptrons.

Given a gradient of $\nabla E(\mathcal{W})$ we adjust the weights $w_{i j}^{k}$ according to the rule

$$
w_{i j}^{k} \leftarrow w_{i j}^{k}+\eta \delta \cdot \pi\left(x_{i j}^{k}\right)
$$

where $\delta$ is the error, $\pi \in \mathcal{I}(X, W)$ is the embedding chosen to evaluate the derivatives $\partial E / \partial w_{i j}^{k}$, and $\pi\left(x_{i j}^{k}\right)$ is the permuted input.

Once we know how a structural perceptron operates, it is straightforward to assemble several structural units with conventional processing units to a structural MLP. For sake of presentation, we restrict to the simple case that structural perceptrons may occur only in the first hidden layer.

A structural single-layer network implementing a function $\boldsymbol{y}=h(X, \mathcal{W})$ with $\boldsymbol{y} \in \mathbb{R}^{m}$ is composed of $m$ structural output units, each associated with an attributed weight graph. A structural multi-layer network with $L \geq 2$ layers is composed of a structural single-layer network implementing the function $\boldsymbol{y}_{\mathbf{1}}=$ $h\left(X, \mathcal{W}_{1}\right)$ and a conventional vectorial network with $L-1$ layers representing the function $\boldsymbol{y}=h_{L}\left(\boldsymbol{y}_{L-1}, \mathcal{W}_{L}\right)$ such that (2) holds for all $l \in\{2, \ldots, L\}$. Since $\boldsymbol{y}_{1}=h_{1}\left(X, \mathcal{W}_{1}\right)$ is a real valued vector, it is straightforward to link the modules $h_{1}\left(X, \mathcal{W}_{1}\right)$ and $h_{2}\left(\boldsymbol{y}_{1}, \mathcal{W}_{2}\right)$ implementing the first and second layer, resp., of the structural MLP. The forward and backward passes, and the error correcting rule for layers $l \geq 2$ follow the same procedure as for vectorial MLPs.

Structural Competitive Learning. Simple competitive learning (CL) is well suited to cluster or categorize unlabeled data points where the competitive network discovers statistically salient features by itself from the correlations of the data points. Competitive learning is closely related to Vector Quantization, Adaptive Resonance Theory, or Self-Organizing Maps. Here, we are primarily concerned with CL as an elementary building block of unsupervised neural learning machines from a conceptual rather than practical point of view.

Clustering $M$ attributed graphs $\mathcal{X}=\left\{X_{1}, \ldots, X_{M}\right\} \subseteq \mathcal{G}_{\mathcal{A}}$ amounts to partition the feature space $\mathcal{G}_{\mathcal{A}}$ such that the average distortion of data graphs to their 
cluster centers $\mathcal{Y}=\left\{Y_{1}, \ldots, Y_{K}\right\} \subseteq \mathcal{G}_{\mathcal{A}}$ is minimized. The average distortion to be minimized is of the form

$$
E(M, \mathcal{Y}, \mathcal{X})=\frac{1}{N} \sum_{j=1}^{K} \sum_{i=1}^{N} m_{i j} \delta\left(X_{i}, Y_{j}\right)
$$

where $\delta($.$) measures the structural distortion induced by the representation Y_{i^{*}}$ of data graph $X_{i}$. Here we assume that the structural distortion $\delta($.$) is the$ Euclidean distance induced by the SH inner product. Then competitive learning proceeds as follows to minimize $E$ in online mode ${ }^{1}$ :

1. Initialize $\mathcal{Y}=\left\{Y_{1}, \ldots Y_{K}\right\}$.

\section{Repeat}

(a) Randomly select a data graph $X \in \mathcal{X}$.

(b) Find $Y_{i^{*}}$ with $i^{*}=\arg \min _{i} \delta\left(X, Y_{i}\right)$ and $\pi \in \mathcal{I}\left(X, Y_{i^{*}}\right)$.

(c) Adjust $Y_{i^{*}}$ by using the update formula $Y_{i^{*}} \leftarrow \eta X^{\pi}+(1-\eta) Y_{i^{*}}$.

(d) Decrease learning rate $\eta$.

3. until no noticeable changes in $\mathcal{Y}$ are observed.

4. Output cluster centers $\mathcal{Y}=\left\{Y_{1}, \ldots Y_{K}\right\}$.

The essential parts of the algorithm are step (2b) and (2c). Step (2b) selects the model $Y_{i^{*}}$ closest to the current data graph $X$ with respect to the Euclidean distance. In step (2c) the competitive learning rule adjusts model $Y_{i^{*}}$ to move it closer to the current data graph $X$. This makes the winner more likely to win on $X$ in the future. Indeed, it has been shown in [7], that any graph isomorphic to $\eta X^{\pi}+(1-\eta) Y$ is a weighted mean of $X$ and $Y$ in the sense of Jiang et al. [8].

Discussion. Besides the well known limitations neural learning machines in the domain of feature vectors, additional problems arise when dealing with graphs.

Elusiveness of adaptive processing: The gradients $\nabla E$ of both error functions as a function of adjustable parameters are not well defined, because they both depend on the the particular choice of an embedding. Consequently, there may be several directions of steepest descent. Hence adjusting the parameters may move the algorithm in a wrong direction. Since there is no canonical embedding the algorithm may have a tendency to zigzag its way through the weight space without gaining substantial improvements. Moreover, the non-uniqueness of $\nabla E$ makes a theoretical analysis of convergence properties difficult.

Computational inefficiency: As opposed to learning on vectorial data, neural learning machines in the domain of attributed graphs are computationally inefficient. The exponential computational effort results from the NP completeness of determining the SH inner product. Solving a large number of NP-complete problems to train a neural network may hinder its practical use. Thus we may resort to approximate solutions of the SH inner product. Approximate solutions, however, increase the number of potential directions to mislead the algorithm. In the case of MLPs, using approximate solutions of the $\mathrm{SH}$ inner product might

\footnotetext{
${ }^{1}$ Minimizing (4) in batch mode corresponds exactly to the K-means algorithm [7].
} 


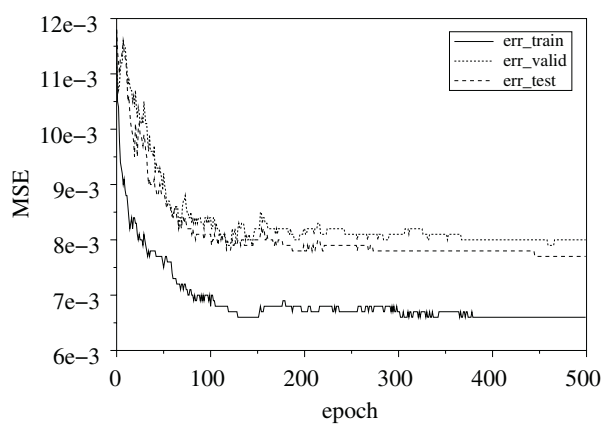

Fig. 1. The evolving MSE of the function regression problem. Shown are the training (solid line), validation (dotted line), and test error (dashed line).

have a similar effect as training with noise. The approximations will smear out data graphs and reduce over-fitting.

Optimal MLP architecture: Due to missing analytical tools, the most natural choice of classifiers in the area of structural pattern recognition are K-nearest neighbor (KNN) classifiers. The performance of KNN classifiers is heavily dependent on the choice of K and the similarity measure. Structural MLPs sweep this problem under a big rug of determining the optimal number of structural and vectorial hidden units and the order of weights graphs in the first hidden layer.

\section{Experiments}

This section serves to illustrate that adaptive processing of structures using structural perceptrons can be successfully applied to simple function regression, classification, and clustering tasks on attributed graphs. In all of our experiments we used an approach proposed by [7] to approximate the SH inner product.

\section{Function Regression}

In our first experiment we tested a structural MLP on a function regression problem. The data set was generated by sampling the function

$$
f: \mathcal{W}_{\mathbb{R}}^{20} \rightarrow[0,1], \quad X \mapsto \frac{\sum_{(i, j) \in E(X)}\left|\mu_{X}(i, j)\right|}{|X|(|X|-1)}
$$

where $\mathcal{W}_{\mathbb{R}}^{20}$ denotes the set of all random weighted graphs $X$ of order $|X| \leq 20$ having weights drawn from a $N(0,1)$ normal distribution. The function $f(X) \in$ $[0,1]$ measures the weighted edge density of a graph $X$.

To compile the data set, we generated 1250 graphs from a uniform distribution over the set $\mathcal{W}_{\mathbb{R}}^{20}$. The data set was divided into a training, validation, and test set composed of 500, 250, and 500 weighted graphs, respectively.

We used a two-layer perceptron consisting of 10 structural units with tanhactivation function and one linear output unit. Each weight graph in the first 

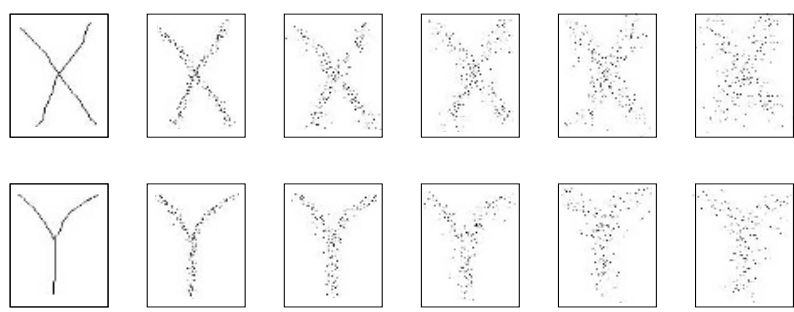

Fig. 2. Images of handwritten characters ' $X^{\prime}$ and ' $Y$ '. The first column shows the model images. Column 2-6 are samples of corrupted images with increasing noise level $\sigma=2,4,6,8,10$. For sake of presentation no rotation is shown.

hidden layer was of order 3 . We set the initial learning rate $\eta$ and the momentum term $\alpha$ to 0.1 . We trained the network using the standard mean sum-of-squares error (MSE) function.

Figure 1 plots the training, validation and test error against the number of passes through the training set. All three error rates have converged by maintaining a small oscillation around $E_{\text {train }}=0.0066, E_{\text {valid }}=0.008$, and $E_{\text {test }}=0.0077$. Since $\eta \rightarrow 0$, the oscillations are due to the randomness of the approximate solution of the SH inner product. From the plot we see that randomness of approximations is similar to training with noise and therefore prevents over-fitting of the training data.

\section{Classification: Synthetic Characters}

In this experiment we investigated the capabilities of a single structural perceptron (SP) to deal with both types of errors occuring in graph based representations, structural variations and noisy attributes.

We used synthetic data to emulate rotation, translation, and scaling invariant handwriting recognition of characters as it typically occurs in pen technology of small hand-held devices. We have drawn two handwritten characters models ' $X^{\prime}$ and ' $Y$ '. The contours of each image were expressed as a set of points in the $2 \mathrm{D}$ plane. For each model we generated corrupted data characters as follows: First we randomly rotated the model image. Then to each point we added $N(0, \sigma)$ noise with standard deviation $\sigma=2,4,6,8,10$. Each point had $10 \%$ probability to be deleted. Figure 2 shows the models and a sample of corrupted data images. Each point set was transformed to a fully connected attributed graph. Vertices represent the points and edges an abstract line between the corresponding points. The attributes are from $\mathcal{A}=[0,1]^{3} \subseteq \mathbb{R}^{3}$ and contain normalized distance values including their statistical spread and location parameters. Table 1 summarizes the structural variation of the data set revealing a strong variation, in particular at a high noise level.

We trained a SP with logistic sigmoid activation function. The weight graph $W$ was of order $|W|=20$. The learning rate was initially set to 0.1 and the momentum term to 0.2 . The network was optimized using the cross-entropy error function. 
Table 1. Structural variations of the character data set. Shown are the mean, variance, and the maximal difference $\Delta=\max -\min$ of vertices for each noise level $\sigma$. For each entry $x / y$ the numbers $x$ and $y$ refer to character ${ }^{\prime} X^{\prime}$ and ' $Y^{\prime}$, respectively.

\begin{tabular}{lccccc}
\hline & 2.0 & 4.0 & 6.0 & 8.0 & 10.0 \\
\hline mean & $16.8 / 20.5$ & $21.7 / 25.9$ & $27.2 / 32.9$ & $31.6 / 38.2$ & $36.0 / 43.3$ \\
var & $0.98 / 1.07$ & $3.70 / 4.10$ & $4.84 / 6.35$ & $6.75 / 7.41$ & $7.43 / 9.40$ \\
$\Delta$ & $6.0 / 6.0$ & $11.0 / 12.0$ & $12.0 / 16.0$ & $17.0 / 14.0$ & $15.0 / 19.0$ \\
\hline
\end{tabular}

Table 2. Classification results: (a) synthetic characters and (b) arm postures. To (a): Shown are the percentage test error rates of the SP and SV classifiers for varying noise level $\sigma$. To (b): Shown are the number of misclassified samples by $\mathrm{NN}_{0}$ and $\mathrm{NN}_{1}$ for each class and the total percentage error rate $E$. The numbers in parenthesis indicate the number of images of the corresponding class.

(a)

\begin{tabular}{lllllr}
\hline$\sigma$ & 2 & 4 & 6 & 8 & 10 \\
\hline $\mathrm{SP}$ & 0 & 0 & 1 & 2 & 2 \\
$\mathrm{SV}_{1}$ & 0 & 0 & 6 & 8 & 29 \\
$\mathrm{SV}_{2}$ & 0 & 0 & 4 & 6 & 23 \\
$\mathrm{SV}_{3}$ & 0 & 0 & 5 & 4 & 29 \\
$\mathrm{SV}_{4}$ & 0 & 0 & 5 & 4 & 20 \\
\hline
\end{tabular}

(b)

\begin{tabular}{rrrrrrr}
\hline & $P_{0}$ & $P_{1}$ & $P_{2}$ & $P_{3}$ & $P_{4}$ & $E$ \\
& $(37)$ & $(48)$ & $48)$ & $(48)$ & $(48)$ & {$[\%]$} \\
\hline $\mathrm{NN}_{0}$ & 3 & 15 & 44 & 40 & 0 & 44.5 \\
$\mathrm{NN}_{1}$ & 3 & 0 & 0 & 1 & 0 & 1.7 \\
\hline
\end{tabular}

We compared the SP classifier with four support vector (SV) classifiers for proximity data: $\left(\mathrm{SV}_{1}\right)$ the pairwise proximities classifier proposed by [5], $\left(\mathrm{SV}_{2}\right)$ the same classifier with $\mathrm{RBF}$ kernel, $\left(\mathrm{SV}_{3}\right)$ support vector learning using the $\mathrm{SH}$ inner product as a non-positive semi-definite 'kernel', and $\left(\mathrm{SV}_{4}\right)$ support vector learning with RBF kernel on the Euclidean distance induced by the SH inner product. To provide a fair comparison we used the same setup as in [3]. We sampled 50 training examples of each character and performed 10-fold cross validation to estimate the generalization error.

The results are given in Table 2(a). The proposed SP algorithm performed more robust to noise and structural variation than the SV classifiers. As expected, the performance of all classifiers decreased with increasing noise level though the recognition rate, in particular for the SP classifier, is very good even for highly corrupted characters.

\section{Clustering: Sensing People}

In our last experiment we applied the CL algorithm to learn the class structure of arm postures as shown in Figure 3. Five different classes of 229 postures are considered: $\left(P_{0}\right)$ Unknown, $\left(P_{1}\right)$ NoArms, $\left(P_{2}\right)$ RightArm, $\left(P_{3}\right)$ LeftArm, and $\left(P_{4}\right)$ BothARms, each referring to the lifted arms of a person. Each image was obtained by automatically localizing a person in video data from a camera. The localized person was enclosed in a bounding box. Position of body parts, like head and hands of the person are identified by skin color. We transformed 


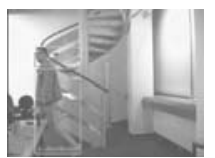

(a) $P_{0}$

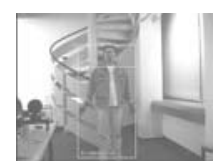

(b) $P_{1}$

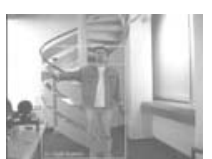

(c) $P_{2}$

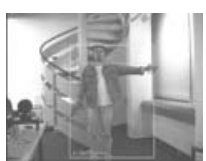

(d) $P_{3}$

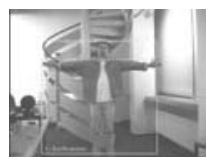

(e) $P_{4}$

Fig. 3. Sample images of arm postures.

each image to a fully connected attributed graph. The vertices represent the left or right corner of the box, or a body part. We used the 1-c coding scheme with $c=3$ to transform the discrete attributes into a numerical vector $\boldsymbol{a} \in\{0,1\}^{3}$. Edge attributes are the distance between the corresponding components in the image.

We randomly selected five perturbed patterns of each class to initialize the models ${ }^{2}$. After 450 iterations the average distortion $E$ has almost converged. Small oscillations of $E$ are due to the approximative nature of the proposed algorithm and the non-uniqueness of the weighted mean. Table 2 shows the percentage error rate of the nearest neighbor classifiers using the models after initialization $\left(\mathrm{NN}_{0}\right)$ and after clustering $\left(\mathrm{NN}_{1}\right)$. The results indicate that structural CL is able to find the class structure of the data set.

\section{Conclusion}

In this paper we considered the problem of constructing supervised and unsupervised neural learning machines when data is given in terms of attributed graphs. We proposed a structural perceptron for adaptive processing of graphs. The key concepts to facilitate learning on graphs are adjustable attributed graphs and the SH inner product of graphs mimicking inner products of vector spaces. In first experiments we applied structural perceptrons to solve supervised and unsupervised learning problems. The main problems, however, with structural perceptrons are the elusiveness of adaptive processing and the high computational complexity. Hence, for practical application the problem of analytical and computational intractability inherent in the SH inner product will be of increasing importance. Possible directions of future work include application of structural perceptrons to other supervised and unsupervised neural learning architectures, experimental validation on practical problems, investigations on tricks of the trade to accelerate convergence, and theoretical analysis on statistical learning theory, representational capabilities and convergence properties.

\section{References}

1. H. Bunke, P. Foggia, C. Guidobaldi, and M. Vento. Graph clustering using the weighted minimum common supergraph. In E. Hancock and M. Vento, editors, Graph Based Representations in Pattern Recognition. 4th IAPR International Workshop, GbRPR 2003, LNCS 2726, pages 235-246. Springer-Verlag, 2003.

\footnotetext{
${ }^{2}$ The initialization scheme is due to the fact that simple CL performs poor even for feature vectors, if the initial models are not chosen carefully.
} 
2. T. Gärtner. A survey of kernels for structured data. SIGKKD Explorations, 5(1):49-58, 2003.

3. P. Geibel, B. Jain, and F. Wysotzki. SVM learning with the SH inner product. In M. Verleysen, editor, Proc. of the 12th European Symposium on Artificial Neural Networks, ESANN'04. D-Facto, Brussels, 2004. Accepted for publication.

4. S. Günter and H. Bunke. Self-organizing map for clustering in the graph domain. Pattern Recognition Letters, 23:401-417, 2002.

5. R. Herbrich. Learning Kernel Classifiers. The MIT Press, Cambridge, MA, 2002.

6. T. Hofmann and J. Buhmann. Pairwise data clustering by deterministic annealing. IEEE Trans. on Pattern Analysis and Machine Intelligence, 19(1):1-14, 1997.

7. B. Jain and F. Wysotzki. Central clustering in the domain of graphs. Machine Learning Journal, 2004. Accepted for publication.

8. X. Jiang, A. Münger, and H. Bunke. On median graphs: properties, algorithms, and applications. IEEE Transactions on Pattern Analysis and Machine Intelligence, 23(10):1144-1151, 2001.

9. M. Lozano and F. Escolano. Em algorithm for clustering an ensemble of graphs with comb matching. In A. Rangarajan, M. Figueiredo, and J. Zerubia, editors, Energy Minimization Methods in Computer Vision and Pattern Recognition, EMMCVPR 2003, LNCS 2683, pages 52-67. Springer-Verlag, 2003.

10. B. Luo, R. Wilson, and E. Hancock. Spectral embedding of graphs. Pattern Recognition, 36(10):2213-2223, 2003.

11. M. V. P. Foggia, R. Genna. Symbolic vs connectionist learning: an experimental comparison in a structured domain. IEEE Transaction on Knowledge and Data Engineering, 13(2):176-195, 2001.

12. A. Sperduti and A. Starita. Supervised neural networks for the classification of structures. IEEE Transactions on Neural Networks, 8(3):714-735, 1997. 